REVISTA ANDALUZA DE ANTROPOLOGÍA.

NÚMERO 12: PATRIMONIOINMATERIAL:REDUCCIONISMOS, CONFLICTOSE INSTRUMENTALIZACIONES. INTANGIBLE CULTURAL HERITAGE: REDUCTIONISMS, CONFLICTS AND INSTUMENTALIZATIONS.

MARZO DE 2017

ISSN 2174-6796

[pp. 144-166]

http://dx.doi.org/10.12795/RAA.2017.12.07

Recibido: 10/06/2016

Aceptado: 07/02/2017

\title{
COMO “COMERSE” UN PATRIMONIO: CONSTRUIR BIENES INMATERIALES AGROALIMENTARIOS ENTRE DIRECTIVAS TÉCNICAS Y EMPRESARIADO PATRIMONIAL
}

\section{HOW TO «EAT» OUR HERITAGE: CONSTRUCTING FOOD HERITAGE BETWEEN TECHNICAL DIRECTIVES AND HERITAGE ENTREPRENEURSHIP}

\section{Chiara Bortolotto}

EHESS-Ecole des hautes Études en Sciences Sociales

Traducción:

Texto original en Italiano traducido por Gema Carrera Diaz. IAPH

\section{Resumen.}

Este artículo explora el creciente dominio del patrimonio cultural inmaterial alimentario en el marco de la Convention de la UNESCO para la salvaguarda del patrimonio cultural inmaterial. El análisis traza la fricción entre los principios y objetivos de la Convención y las expectativas iniciales de los primeros proyectos de patrimonialización en la esfera alimentaria. A partir de la observación etnográfica de la producción del expediente de la "Comida gastronómica de los franceses" se revisará el proceso de adecuación de los proyectos locales al dispositivo internacional poniendo así al día el poder performativo de las directivas técnicas elaboradas a menudo informalmente para alinear las solicitudes de los Estados con las prioridades de la Convención. Esta experiencia técnica permite separar los aspectos culturales dentro del sector de la alimentación de otras facetas, incluyendo la comercial, no obstante orgánicamente relacionados con este campo. Si los documentos presentados a la Unesco se esfuerzan por eliminar estos temas polémicos, siguen siendo el centro de las preocupaciones de los actores sociales y políticos, y se encuentran actualmente en el centro del debate sobre la relación entre la salvaguardia 
del patrimonio cultural inmaterial y el desarrollo sostenible puestos en cuestión por las implicaciones del uso neoliberal de la cultura como un recurso.

\title{
Palabras Claves.
}

UNESCO, Patrimonio Cultural Inmaterial, alimentos, directivas técnicas, gobernanza global, mercado.

\begin{abstract}
.
This article explores the field of food-related intangible cultural heritage in the framework of the Unesco Convention for the Safeguarding of the Intangible Cultural Heritage. The analysis outlines the frictions between the Convention's principles and objectives and the initial expectations of the first heritage projects in the food realm. Grounded on the ethnographic observation of the making of the nomination file of the «Gastronomic meal of the French», the article examines the complex encounter between local priorities and endeavours on one side and international norms and procedures on the other. The tension arising from this encounter sheds light on the agency of technical directives informally put forward by international organisations to harmonise the States' requests within global governance apparatuses. In our case technical expertise isolates foodways' cultural aspects from other features, in particular commercial interests, despite their organic integration within this field. Even if the nomination files submitted to Unesco try to clear these controversial matters, the latter stay high among the concerns of social and political actors. Currently at the core of the raising debate on the relationship between the safeguarding of intangible cultural heritage and sustainable development within Unesco, ICH's economic potential question the implications of the neoliberal uses of culture as a resource.
\end{abstract}

\section{Keywords}

UNESCO, Intangible Cultural Heritage, Foodways, technical directives, global governance, market.

\section{PATRIMONIO IMMATERIAL Y PRÁCTICAS ALIMENTARIAS ${ }^{1}$}

"Como comerse un patrimonio mundial" es el nombre del proyecto surgido en Matera ${ }^{2}$, “ciudad de las piedras", inscrita en la Lista del Patrimonio Mundial desde 1993 y área de

1. Esta investigación ha sido llevada a cabo, en una primera fase en el ámbito del proyecto EAT (Etnografíe alimentari transfrontaliere) apoyado por el Fundación suiza Pro Helvetia y posteriormente desarrollado en el marco del proyecto "UNESCO frictions: Heritage-making across global governance" -ANR-14-ACHN- 0006-01) gracias a una generosa financiación de la Agence Nationale de la Recherche. Agradezco a Gema Carrera Díaz por la traducción del original en italiano.

2. Matera es una ciudad de la Basilicata convertida, entre los años 40 y 50, en símbolo del atraso y del subdesarrollo del sur de Italia". Ello se debe también a la descripción que Carlo Levi hizo de ella en Cristo si è fermato ad Eboli (1945). 
producción del "pan de Matera", desde 2006 tutelado por la marca de calidad europea "indicación geográfica protegida". En la convicción de que "observar un sitio de la UNESCO es un gesto nutritivo, ya sea figurado o literal", dos promotores culturales locales $^{3}$, conscientes del hecho de que conocer y degustar los productos del territorio es ya parte integrante de la aventura patrimonial, siempre más enfocada al lado experiencial (Fabre, 2016: 55), proponen asociar un alimento considerado representativo de la identidad local, el pan de Matera, a la valorización del sitio. El proyecto se extiende a todos los sitios italianos de la Unesco para "hacerlos utilizables en todos los sentidos" asociando, por ejemplo, el limoncello a la costa de Amalfi, la pizza a Nápoles, la leche de almendras a los Trullos de Alberobello, el queso de oveja a Pienza, el pesto a Génova (Grillo, 2008).

Con la introducción de la categoría de patrimonio inmaterial, las prácticas alimentarias adquieren una legitimidad patrimonial por sí misma. ¿De qué manera se han convertido en patrimonio cultural inmaterial reconocido por la UNESCO? ¿De qué modo la necesidad de inscribirse en el ámbito de las políticas internacionales establecidos por esta Organización produce nuevas representaciones de estas prácticas? ¿Cómo viene estructurada la dimensión comercial, inherente a estos elementos, con la patrimonial, por definición ajena a la lógica del mercado? ¿En qué modo el reconocimiento de este nuevo ámbito partrimonial influye sobre el propio concepto de "bien cultural"? Para responder a estas preguntas me voy a basar en la observación etnográfica de situaciones e instituciones patrimoniales situadas en una multiplicidad delugares y en diferentes niveles de la escala de la gobernanza global del patrimonio cultural inmaterial: la Comisión Nacional Italiana para la UNESCO; las reuniones del Comité Intergubernamental para la salvaguardia del patrimonio cultural inmaterial"; las del Comité de expertos que acompañaron a la preparación de la candidatura francesa de "Comida gastronómica de los franceses” entre 2007 y 2010; la reunión de expertos que, en 2008, se refirió a la relación entre los hábitos alimentarios y el alcance de la intervención de la Convención para la salvaguardia del patrimonio cultural inmaterial (UNESCO, 2003). En el siguiente análisis voy a explorar la construcción contemporánea del campo del patrimonio cultural inmaterial alimentario en el ámbito de la aplicación de la Convención de la UNESCO sobre el fondo del fortalecimiento de la compleja y ambigua relación entre el patrimonio y el desarrollo sostenible.

3 Agradezco a Antonio Nobile e Dino Scote por haber compartido conmigo su proyecto.

4. El Comité Intergubernamental para la salvaguardia del patrimonio cultural inmaterial se compone de veinticuatro Estados partes en la Convención, elegidos por un período de cuatro años, según el principio de representación geográfica equitativa y de rotación. Las sesiones del Comité duran sobre cinco días y cada año ejerce de anfitrión uno de sus miembros. Durante los últimos ocho años he observado las sesiones organizadas en Abu Dabi (2009), Bali (2011), París (2012 y 2014), Bakú (2013), Windhoek (2015) y Addis Abeba (2016) y he seguido la organizada en Nairobi mediante podcast (2010). 


\section{DE LA PIZZA AL “ARTE DE LOS PIZZEROS NAPOLITANOS”}

Utilizar la Convención para la salvaguardia del patrimonio cultural inmaterial para construir patrimonio alimentario es una idea a la que los padres fundadores de este instrumento se opusieron fuertemente en los primeros años de su funcionamiento, desde la aprobación por la Conferencia General de la UNESCO en el año 2003 (Cang, 2015: 49). De hecho, el interés de incluir las prácticas alimentarias en el ámbito del patrimonio inmaterial precede a la Convención. Ya desde 2001 el entonces Ministro de Agricultura italiano, Alfonso Pecoraro Scanio, había propuesto reconocer la pizza como una obra maestra del patrimonio oral e inmaterial de la humanidad ${ }^{5}$. El proyecto del Ministro levantó encendidas polémicas que, gracias a unas prácticas realizadas en este mismo período en la Comisión Nacional para la UNESCO, he podido observar de cerca: la Comisión fue literalmente asaltada por los periodistas que, junto con la propuesta del Ministro, descubrieron el concepto de patrimonio inmaterial, siendo en gran medida desconocido para la mayoría. La perplejidad y la ironía provocada por esta iniciativa han tenido, por tanto, el efecto de la introducción del concepto de patrimonio inmaterial en la prensa nacional e internacional. Este concepto, sin haber entrado aún en el vocabulario común, ha resultado difícil tanto para los profesionales como para los no especialistas y la propuesta de considerar la pizza como un activo cultural inmaterial había ofrecido un punto de partida para preguntarse sobre la expansión de las fronteras del concepto de patrimonio. Un artículo titulado "civilización inmaterial", publicado en The Atlantic Monthly (Murphy, 2001) y luego citado por los primeros trabajos académicos sobre la nueva categoría introducida por la UNESCO (Kirshenblatt-Gimblett, 2004: 56; Hafstein, 2008: 98), toma como ejemplo precisamente la propuesta del ministro italiano de denunciar lo que al periodista le parecía un concepto sin sentido, lo que podría haber puesto en voz pasiva, la dilación o amnesia selectiva. Estas críticas del sentido común tenían, y aún tienen, las correspondientes críticas en el ámbito académico: “inflación patrimonial" (Jeudy, 2001; Heinich, 2009) o “tout patrimoine" (Hartog, 2003) son sólo algunas de las expresiones que se propagan en los últimos años para indicar expansión sin límites de la esfera patrimonial (Lowenthal, 1996).

A pesar de su éxito mediático, la propuesta de 2001 no fue llevada a cabo. Sin embargo, una nueva solicitud de candidatura a la lista representativa del patrimonio cultural inmaterial de la humanidad figura, desde 2012, en la lista de espera elaborado por la Secretaría de la UNESCO con el título de "arte tradicional de los pizzeros napolitanos". Tal propuesta se hizo oficial por parte del Consejo directivo de la Comisión Nacional Italiana para la UNESCO y, si el Comité Intergubernamental para la salvaguardia del

5. Este programa estuvo activo entre 2001 y 2005, antes de la entrada en vigor de la Convención (Nas, 2002).

6. El atributo "tradicional" ha sido retirado del nombre del elemento en la solicitud enviada en 2015. 
patrimonio cultural inmaterial decide inscribir este elemento en la Lista Representativa, "El arte de los pizzeros napolitanos" se convertirá en uno de los elementos italianos que representan el patrimonio inmaterial de la humanidad.

Las nuevas prioridades de la política italiana del patrimonio inmaterial reflejan una evolución más generalizada de la aplicación de la Convención para la salvaguardia del patrimonio cultural inmaterial. El creciente número de registros de prácticas relacionadas con el ámbito alimentario en las listas establecidas por la Convención ha dado a estas últimas una legitimidad que no tenían cuando el concepto de patrimonio inmaterial fue introducido en la escala internacional a principios del 2000. Numerosos elementos hoy inscritos en las listas internacionales establecidas por la Convención de la UNESCO para la salvaguardia del patrimonio cultural inmaterial tienen, de hecho, un vínculo más o menos directo con el ámbito agroalimentario. La comida gastronómica de los franceses, la cocina tradicional mexicana y la dieta mediterránea, inscritas en el $2010^{7}$, han allanado el camino para muchas otras candidaturas ${ }^{8}$ alimentando un debate que ha explicado los aspectos más controvertidos de tales iniciativas permitiendo dirigir los proyectos sucesivos de una manera considerada más en línea con el espíritu de la Convención.

\section{LA GASTRONOMÍA FRANCESA}

En noviembre de 2010, el Comité Intergubernamental para la Salvaguardia del patrimonio cultural inmaterial (PCI), que se reunió en Nairobi, inscribió "la comida gastronómica de los franceses" en la Lista representativa del PCI de la humanidad. Acaloradas controversias han acompañado el proyecto hasta el último momento. Normalmente, de hecho, las delegaciones de los 24 estados miembros del comité intervienen a propósito de la inscripción de los elementos candidatos sólo si la opinión de los órganos de evaluación es negativa. Como evidencia de la naturaleza controvertida de este informe, un delegado japonés intervino, sarcásticamente, para pedir a la delegación francesa una aclaración a pesar de la opinión favorable del órgano evaluador. El delegado de Japón explicó que cuatro restaurantes franceses en Japón han recibido tres estrellas en la guía Michelin y solicitó una aclaración en cuanto a su legitimidad para compartir este reconocimiento y del uso del emblema de la Convenció (UNESCO, 2011: 40-41). Este comentario plantea dos cuestiones centrales: la de la delimitación de la comunidad que reconoce este elemento como un factor identitario- indicada en la candidatura como el "pueblo francés en su conjunto" (UNESCO, 2010: 2), delimitación considerada por muchos como vaga

7. Según los expertos croatas con los que he discutido esta candidatura durante una reunión de expertos organizada por la UNESCO, el arte del pan de especias de Croacia del norte, inscrito también en 2010, no se produce para ser consumido y, de hecho, se incluye en el ámbito de la artesanía.

8. La dieta mediterránea se candidó originalmente por parte de España, Italia, Grecia y Marruecos en 2010. En los años siguientes se han asociado con la candidatura de Chipre, Croacia y Portugal. 
o arbitraria (Tornatore, 2012), y la de las implicaciones comerciales de esta candidatura, dadas las evidentes repercusiones que tal reconocimiento podría tener en el sector de la restauración. Ambos problemas han atormentado a los responsables de esta empresa y han sido para ellos objeto de reflexión cuidadosa.

La participación en el comité científico de la candidatura de lo que se ha dado a llamar la "comida gastronómica de los franceses" (2007-2010) me ha permitido compartir estas preguntas y participar en esta reflexión, a raíz de la fabricación de un elemento del patrimonio cultural inmaterial la humanidad y observar de cerca la encendida polémica generada por la aplicación propuesta.

En 2007, el director del Institut Européen d'Histoire et de Cultures de l'Alimentation (IEHCA), Francis Chevrier, me invitó a participar en el proyecto de candidatura "dados mis conocimientos sobre el patrimonio cultural inmaterial" implicándome en el comité de expertos para "reflexionar sobre la definición de un patrimonio culinario francés y delimitar el perímetro" y encargado del "contenido intelectual y científico del expediente" L'IEHCA creado en el seno de la Universidad François Rabelais de Tours y acababa de lanzar la idea de la candidatura y comenzó a presionar a escala nacional. Una asociación, la Mission française du patrimoine et des cultures alimentaires (MFPCA), fue creada para preparar el expediente y presionar a los organismos competentes en vista de la candidatura (IEHCA, s.d.) con el objetivo de "divulgar esta iniciativa entre los franceses"10 para acompañar estos órganos se creó un comité de apoyo y un comité de expertos. El primero, compuesto por los chefs con estrellas y personalidades de la política, del arte y de los medios de comunicación y la industria, así como de organizaciones como el Cordon Bleu, Slow Food, o l'Association des Sites remarquables du gout, tenía la tarea de "apoyar públicamente este proyecto" y "solicitar a los actores locales". El segundo, formado por académicos e intelectuales de los especialistas en historia y culturas alimentarias se encarga, como se mencionó anteriormente, de "la elaboración de la sección científica del dossier de la candidatura" y "definir con precisión el perímetro del patrimonio alimentario francés" (IEHCA, s.d.).

Durante la primera reunión del Comité de expertos, cuando todavía nadie de los presentes estaba familiarizado con los principios fundamentales de la Convención de la UNESCO, el director del IEHCA ha explicado cómo esta institución se había ya dirigido a los Ministerios de Agricultura, de Turismo y estuvo procurando el apoyo del ministerio de la Identidad nacional y de las PYMES y cómo ya se habían realizado los primeros contactos con el sector privado e industrial, sectores que habían mostrado un gran

9. Cartra di Francis Chevrier del 16 de febrero de 2007 conservada en los archivos de la autora.

10. Notas de campo. 
interés en este programa. Con evidente satisfacción había llegado a la conclusión de que la ventaja de este proyecto fue que se apoyaba en un sector privado de gran peso ligado no sólo a la industria alimentaria, sino también al turismo ${ }^{11}$.

Los puntos de partida de esta candidatura abiertamente ostentados por sus promotores han minado la recepción de este proyecto en el seno de la UNESCO, donde se temía que este tipo de iniciativas terminaran por sotoponer la Convención para la salvaguardia del patrimonio cultural inmaterial (UNESCO, 2003) a la Convención de dos años después, sobre la protección y promoción de la diversidad de las expresiones culturales (UNESCO, 2005), o sea, que el primer instrumento fue utilizado comercialmente para promover el patrimonio alimentario, intrínsecamente ligado al mercado de la industria agroalimentaria y el catering. Por un lado, como he podido observar durante la participación en una reunión de expertos sobre la interacción entre las dos Convenciones arriba citadas en $2008^{12}$, aunque la Secretaría de la UNESCO puede hacer más para "hablar con una sola voz"13 y coordinar estas herramientas, las interacciones en la aplicación de los dos convenios es extremadamente limitada en el plano institucional. Por otro lado, el discurso de la UNESCO excluye los productos alimenticios y su protección del ámbito de intervención de la Convención, a pesar de que estos representan el resultado de un proceso cultural que le da un sentido de identidad en las comunidades, los grupos e individuos ${ }^{14}$.

A estas dificultades se sumó el error del entonces Presidente de la República, Nicolas Sarkozy, que, en febrero de 2008, en el Salón de la Agricultura, anunció la intención de Francia de reconocer la gastronomía francesa como patrimonio inmaterial de la UNESCO:

"La agricultura y la artesanía están igualmente tras de la diversidad gastronómica de nuestro país. Retengo que Francia es el primer país en presentar, antes de 2009, una solicitud a la UNESCO para el reconocimiento de nuestra herencia

11. Compte-Rendu de la réunion du Conseil d'experts. Projet d'inscription du patrimoine alimentaire français sur la liste du patrimoine culturel immatériel de l'UNESCO, 29 juin 2007-07-04..Archivo personal de la autora.

Expert meeting on interactions between the Convention for the Safeguarding of the Intangible Cultural Heritage (2003) and the Convention on the Protection and Promotion of the Diversity of Cultural Expressions (2005). 31 March - 1 April 2008 UNESCO, Paris.

12. Notas de campo.

13. Para una lectura diferente de la relación entre la Convención del 2003 y la del 2005 vease Csergo 2016.

14. http://www.expatica.com/fr/news/Slow-Food-guru-rubbishes-French-cuisines-heritage$\underline{\text { bid_112296.html }}$ 
gastronómica en el patrimonio mundial. Tenemos la mejor gastronomía en el mundo - en fin, desde nuestro punto de vista ... así sin querer compararnos con otros - queremos que sea reconocida como Patrimonio de la Humanidad. “(Sarkozy, 2008 citado en Tornatore, 2012)

Las críticas recibidas por esta iniciativa se han articulado en diferentes niveles. Después de la declaración del presidente Sarkozy la opinión pública se indignaba del complejo de superioridad francesa: Carlo Petrini, fundador y portavoz del movimiento slow-food se preguntaba sacásticamente por qué la gastronomía francesa debería considerarse mejor que cualquier otra ${ }^{15}$. Otras voces se preguntaban sobre la relevancia de considerar inmaterial la esfera de la nutrición o sobre los límites de este elemento ya que, según las encuestas de opinión, el cuscús sería el plato más común en Francia. En el seno de la UNESCO los obstáculos a esta candidatura se realizaron en términos técnicos o políticoinstitucionales, es decir, por una parte, el elemento era demasiado vasto y, por tanto, la comunidad era difícil de identificar y las medidas de salvaguardia difícilmente aplicables. Por otro lado, el aura elitista de la gastronomía francesa traiciona los principios, no escritos pero profundamente arraigados en la historia de la Convención, que la entienden como una herramienta destinada principalmente a los países del sur "ricos en tradiciones", en contraste con lo occidentales, ya beneficiados de otros dispositivos como el Patrimonio Mundial e implícitamente considerados depositarios de arte y "alta cultura"16.

La seguridad y el optimismo de la primera reunión del comité de expertos habían dado paso, en las siguientes reuniones, al nerviosismo y a las preocupaciones: el caballo de batalla de los promotores de la candidatura se había demostrado incompatible con el espíritu de la Convención, y esto socavó las mismas bases del proyecto. Por consiguiente, era necesario cambiar radicalmente la perspectiva. Como admite el director del IEHCA: "no es este el espíritu con el que hay que abordar la Convención"17. No sólo le parece ahora necesario abordar la impresión negativa que el proyecto francés hizo a la comunidad internacional: "Debemos avanzar con gran humildad ", sino también adaptar el enfoque inicial del proyecto al espíritu de la Convención: "hay que situarse en la perspectiva de l'UNESCO"18. Para orientar este cambio de perspectiva la clave fue una reunión de expertos organizada en Francia por el Ministerio de Cultura y por un agente asociativo

15. La exclusión de expresiones culturales inmateriales occidentales que reflejan la cultura "alta", que para algunos países son incompatibles con el concepto de PCI, termina sin embargo por perpetuar una división entre "Occidente y el resto" en modo no coherente con los objetivos propios de la Convención (Kirshenblatt-Gimblett 2004: 57; Cang 2015).

16. Traducido del francés

17. Notas de campo. Traducido del francés.

18. Conclusions of the group of experts concerning foodways as an integral part of cultural systems and intangible cultural heritage (Vitré, 4 and 5 April 2009). Archivio personale dell'autrice. 
francés muy activo en el ámbito de aplicación de la Convención, la Maison des cultures du monde.

\section{CÓMO CONSTRUIR UNA CANDIDATURA SOBRE ALIMENTACIÓN}

La urgencia de abordar la cuestión candente de candidaturas alimentarias, que estaba empezando a afectar a varios Estados, había surgido durante la sesión del Comité Intergubernamental para la Salvaguardia del PCI en Estambul en 2008. En respuesta a una solicitud del Perú, que preparó la candidatura de su cocina (Matta, 2016), Francia ofreció a acoger una reunión sobre este tema (UNESCO, 2009: 38-39). En 2009, un grupo de expertos internacionales fue invitado a Vitré, en Bretaña, para reflexionar sobre la relación entre las prácticas alimentarias y la esfera del PCI. Las personalidades invitadas fueron algunas de las figuras que más habían contribuido a la reflexión y el debate que acompañó la creación de la Convención en el seno de la UNESCO. El ambiente era aquel, cómplice y amigable, de una reunión entre colegas de toda la vida unidos por un interés común. Yo era una de los pocos que asistieron a esta reunión, junto a la directora científica de la candidatura de la gastronomía francesa.

Las conclusiones de esta reunión confirman la pertinencia de considerar las prácticas alimentarias como una expresión del patrimonio cultural inmaterial si se entienden como experiencias culturalmente elaboradas por las comunidades, y no como una simple respuesta a necesidades biológicas. De acuerdo con los expertos presentes en esta reunión, para ser consideradas "patrimonio inmaterial” las prácticas alimentarias deben entenderse como un proceso orgánico, estructurado y complejo, que vaya desde la elaboración de las materias primas hasta su consumo. Otro de los requisitos es la mejora del carácter transversal a los diferentes aspectos del patrimonio inmaterial: tradiciones y expresiones orales, las prácticas sociales, rituales y actos festivos, conocimientos y prácticas relacionados con la naturaleza y el conocimiento asociado a la artesanía tradicional. En línea con el enfoque participativo de la Convención, que atribuye a las comunidades locales la tarea de reconocer el valor patrimonial de sus prácticas culturales y de participar activamente en su protección, los expertos hicieron hincapié en la importancia de la participación de la comunidad en el proceso de patrimonialización de tales elementos. Por último, dada la importancia de la movilidad y del uso compartido de tales prácticas a través de las fronteras, las recomendaciones de los expertos han puesto el acento en el hecho de que insistir en que éstas radican en un determinado territorio no debe ser la única manera de representarlas ${ }^{19}$. Insistiendo sobre los procesos sin hacer ninguna referencia a los productos, tales conclusiones establecen así un marco que permite representar las prácticas alimentarias como expresiones del patrimonio cultural inmaterial e inscribirlas

19. Note argumentaire pour l'inscription du patrimoine alimentaire français sur la liste représentative du patrimoine culturel immatériel de l'UNESCO, s.d. 
de una manera consistente en el ámbito de intervención de la Convención ocultando debajo de la alfombra las implicaciones económicas intrínsecamente asociadas a este tipo de prácticas.

\section{DE LA “GASTRONOMÍA FRANCESA" A LA “COMIDA GASTRONÓMICA DE LOS FRANCESES"}

El caso francés es un excelente ejemplo del impacto de estas directivas en la representación de lo que fue inicialmente identificado con la "gastronomía francesa". El expediente de la candidatura propuesta a la UNESCO es, de hecho, el resultado de una serie de reescrituras que siguieron a un cambio de perspectiva que concluyó con la candidatura de una comida festiva y ritualizada. Este cambio de perspectiva ha afectado a muchos aspectos que los promotores de la candidatura habían proyectado poner en el centro de la misma. La primera se refiere a la idea de excelencia, en las expectativas de los promotores, debería haber caracterizado el elemento candidato. Para estos últimos, un reconocimiento de la UNESCO evocaba inevitablemente la idea de "valor universal excepcional", base del más conocidos de los dispositivos de esta organización: la lista del patrimonio mundial. Desde esta perspectiva, el objetivo de este trabajo era "hacer reconocer oficialmente por el organismo mundial competente que la cocina francesa es un monumento de la cultura mundial" ${ }^{20}$.

Comolo demuestrala reacción del Presidente de MFPCA, Jean-Robert Pitte, decepcionado y sorprendido por las aclaraciones sobre los principios de la Convención para la salvaguardia del PCI recibidos durante una reunión del Comité de Expertos, el enfoque profundamente relativista de la Convención era pues en principio incomprensible: "si no se habla de la excelencia ni siquiera vale la pena hablar sobre el patrimonio"21.

Si ha sido posible, renunciar a reclamar la excelencia de la gastronomía francesa, la cuestión más espinosa seguía siendo la de asociar este elemento a una comunidad, en consonancia con la definición de PCI acatada por la UNESCO: "se entiende por "patrimonio cultural inmaterial "los usos, representaciones, expresiones, conocimientos y técnicas -junto con los instrumentos, objetos, artefactos y espacios culturales que les inherentes- que las comunidades, los grupos y, en algunos casos, los individuos reconozcan como parte de su patrimonio cultural "(UNESCO, 2003 art. 2.1, énfasis del autor). Por un lado esto chocaba con las posiciones ideológicas que habían dado lugar a la candidatura, como se deprende de las observaciones del Presidente de MFPCA, para quien asociar la gastronomía francesa a una comunidad iría en contra del concepto de universalidad y por lo tanto sería inaceptable ${ }^{22}$. Por otro lado, definir la comunidad de la gastronomía

20. Notas de campo.

21. Notas de campo.

22. Ministère de l'Immigration, de l'Intégration, de l'Identité nationale et du Codéveloppement, creado en 2007 bajo la presidencia de Nicolas Sarkozy. 
francesa era una empresa particularmente difícil como admitió, desanimado, el mismo director de del IEHCA « esto es indefinible, habrá que encontrar algo que no será jamás del todo preciso". Afiliación administrativa; delimitación geográfica; uso de la lengua francesa; criterio de la nacionalidad son algunas de las propuestas que surgieron durante las discusiones del comité de expertos para definir esa comunidad. Criticado dentro del mismo comité que se preguntaba, por ejemplo, cómo definir la relación entrela comunidad musulmana en Francia y la gastronomía francesa que incluye alimentos y bebidas que no son parte de la dieta de los musulmanes practicantes. Estas propuestas han planteado el misma "rompecabezas de la identidad nacional "que unos años antes había sido objeto de un intenso debate a raíz de la creación de un ministerio encargado de la identidad nacional”23 (Detienne, 2010). No sólo, como en el caso de la dieta mediterránea (Marques da Silva, 2016), el perímetro de la comunidad era difícil de definir sino que el director del IEHCA ahora era consciente de que era necesario demostrar a la UNESCO que esta comunidad había dado su consentimiento y que había participado activamente en el proyecto de la candidatura: "debemos organizar el consentimiento, o más precisamente, la participación de la comunidad" ${ }^{24}$. En primer lugar era necesario demostrar que una comunidad reconocía como un elemento identitario la comida gastronómica. Una investigación dirigida sobre una muestra de 998 personas por el Centre de Recherche pour létude et l'observation des modes de vie (CREDOC) ha demostrado que el 95,2\% de los franceses consideran esta práctica como parte de su patrimonio. La participación de la comunidad se justificó mediante la demostración de la participación en el proyecto de candidatura de organismos e instituciones considerados representativos de las comunidad en cuestión, o sea del conjunto del pueblo francés: el IEHCA y la MFPCA son presentados como entidades representativas de esa comunidad. Círculos culinarios, universidades, el Senado, el Institut de France, son también organismos citados como representativos de la totalidad del pueblo francés e involucrados en el proceso de la candidatura.

Los largos debates que acompañaron la elección del nombre del elemento son ejemplos de la reversión de los argumentos presentados en apoyo a la inscripción. Como explica Francis Chevrier presentando en Le Monde su libro de Notre gastronomie est une culture (Chervier, 2011b), candidar a la comida gastronómica, o lo que es lo mismo, una práctica social y popular de interés antropológico, en lugar de la gastronomía, permitió eludir el tema de las referencias a productos que habrían hecho pensar sobre el uso comercial de este proyecto, y el saber hacer culinario de cuyo reconocimiento se beneficiarían los chefs, famosos y no.

23. Notas de campo.

24. La Confrérie du cèpe dans la galaxie UNESCO (Sud-Ouest, 30/12/2010, online: http://www.sudouest.

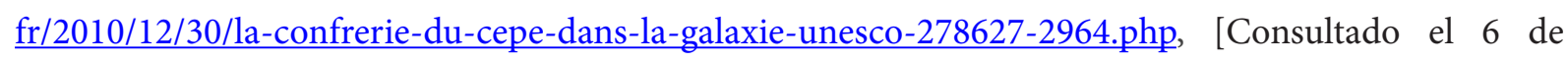
diciembre 2016]. 
No sólo se identificó un aspecto no controvertido y en consonancia con la definición de PCI, sino que además éste se presentó con el fin de cumplir los cinco criterios de selección (Csergo, 2011). La candidatura se moldea con un discurso lo más coherente posible con el espíritu de la Convención de la UNESCO, insistiendo en los beneficios intangibles de dicha empresa, así como en el orgullo que surge del prestigioso reconocimiento por las propias prácticas cotidianas, reflejo de la cohesión social y de una cultura del compartir (Csergo, 2011: 18).

El uso de un vocabulario y de los argumentos apropiados en la construcción del expediente ha determinado el éxito del proyecto francés. Sin embargo, en un contexto de crisis en el que, según la prensa especializada, Francia se convertiría en la segunda plaza de McDonald en términos de ventas en todo el mundo (Steinberger, 2009), las expectativas de beneficios materiales derivados de la inscripción en la Lista Representativa del PCI de la humanidad, son aún los más comunes entre los que ven este iniciativa desde una perspectiva más amplia: "una oportunidad para el comercio exterior (...) 1,3 millones de puestos de trabajo" (Pierre Lellouche, Secretario de Estado de Comercio Exterior, citado en Csergo, 2011: 17).

La utilización económica de dicha empresa era previsible y se daba por descontado, así como el uso simbólico y legitimador del nombre de la UNESCO asociado a la esfera de la gastronomía por parte de los productores o promotores de productos específicos. Tanto los actores locales como las políticas nacionales obtendrán provecho de la inscripción de la comida gastronómica de los franceses. Entre muchos otros ejemplos de estos usos realizados por los actores locales se encuentra el título triunfante que apareció en la prensa regional para anunciar la "entrada de la hermandad de las setas boletus en la galaxia UNESCO"25. A nivel nacional otras intervenciones han seguido al reconocimiento de la comida gastronómica de los franceses, como el proyecto de la creación de una Ciudad de la Gastronomía, presentado por Francis Chevrier como un "escaparate francés de la excelencia" (Chevrier, 2011a: 145) o el lanzamiento , en febrero de 2011, de la campaña So French so Good diseñada para promover los productos agroalimentarios franceses en el extranjero, gracias, entre otras cosas, a un plan de comunicación que ofrece aplicaciones digitales que permiten "encontrar el mejor queso francés en Estambul o croissants en Shanghai” ${ }^{26}$. Incluso sin consultar a la atenta mirada del etnólogo, es evidente que la verdadera promocionada es la gastronomía francesa y no la práctica social de la comida gastronómica y que la UNESCO realiza, aunque incorrectamente, la función de la marca de excelencia (Tornatore, 2012) ${ }^{27}$. Como se ha señalado Clare Sammels en el caso

25. http://archives.gouvernement.fr/fillon_version2/gouvernement/campagne-so-french-so-goodencourager-la-gastronomie-francaise-a-s-exporter.html, [Consultado el 7 de desembre 2016].

26. Significativamente, el "repas gastronomique des Français" ha sido realmente registrado como una marca por el MFPCA.

27. La influencia de las decisiones estratégicas tomadas por los expertos franceses habrían terminado por reafirmar la primacía de la gastronomía francesa, ejerciendo una "violencia simbólica" sobre el imaginario de los países que han aceptado tácitamente inspirarse en su ejemplo (Cang 2015). 
mexicano, el reconocimiento dado a las prácticas sociales y domésticas termina por promover los productos locales asociados con ellas en el mercado internacional (2014).

Si un análisis antropológico de las dinámicas que han acompañado el registro de la "comida gastronómica de los franceses" sirve no tanto para denunciar el uso comercial fácilmente predecible de un dispositivo de este tipo, ya ampliamente documentado por la prensa y ante los ojos de cualquier observador atento, éste permite explorar los desarrollos contemporáneos del dispositivo patrimonial. Este expediente es particularmente adecuado para preguntarse acerca de las nuevas articulaciones de dichos usos con el concepto de patrimonio, históricamente fundamentado en la idea de la inalienabilidad, y para reflexionar sobre cómo la dimensión comercial del patrimonio modifica nuestra representación de la cultura y de sus funciones. Además, este ejemplo permite ilustrar el papel de las directivas técnicas en la gobernanza internacional.

\section{EL AUGE DE LAS CANDIDATURAS ALIMENTARIAS: EL PODER PERFORMATIVO DE LAS DIRECTIVAS TÉCNICAS Y LA CUESTIÓN DEL MERCADO}

El análisis de los expedientes de solicitud de candidaturas en los años siguientes a la reunión de expertos de 2009 muestra cómo las recomendaciones de Vitré, los comentarios técnicos de la Secretaría de la Convención sobre las solicitudes recibidas, las enseñanzas impartidas por una red de facilitadores formados por la Secretaría para llevar a cabo intervenciones de "capacitación", o los consejos de ex funcionarios de la UNESCO o de otros expertos familiarizados con la práctica de la Convención y empleados como consultores en la preparación de candidaturas han sido aprovechados para obtener beneficios en el desarrollo de candidaturas que presentan las prácticas alimentarias insistiendo sobre los aspectos culturales (Cang, 2015, Matta, 2016, Csergo, 2016, Marques da Silva, 2016).

Una gran diversidad caracteriza a los elementos inscritos. Los primeros se presentan como prácticas y culturas culinarias o sistemas alimentarios como un todo: además de la comida gastronómica de los franceses, la dieta mediterránea y la cocina tradicional mexicana. Japón ha elegido nominar las tradiciones culinarias japonesas, registrados en 2013, como un sistema alimentario, insistiendo en su relación con una práctica festiva (Cang, 2015).

Otros elementos se describen como eventos rituales en los cuales las prácticas alimentarias juegan un papel importante. Es el caso del expediente de Serbia en relación con la celebración de la fiesta del santo patrón de la familia (Slava), registrado en 2014. La "tradición ceremonial de keşkek", presentado por Turquía y registrada en 2011, es la preparación de un plato de trigo y carne. Esta práctica es descrita insistiendo sus 
funciones sociales y rituales subrayando la participación, con roles específicos de los diferentes actores de la ceremonia con motivo de acontecimientos que marcan la vida personal, como bodas o circuncisiones, o social, tales como fiestas nacionales y religiosas.

Algunas inscripciones relativas a la esfera de los alimentos están relacionadas con prácticas agrícolas, tales como la tradición vitivinícola en kwevri (grandes tinajas semienterradas) inscrita en 2013 a propuesta de Georgia. Otras tres prácticas agrícolas han sido reconocidas en 2014, respectivamente, por Italia, Grecia y Marruecos: el cultivo de la vid retoño de las comunidades de Pantelleria; los saberes del cultivo de "mastic" de las Isla de Quíos y los conocimientos vinculado con el árbol de argán en Marruecos. Incluso en las candidaturas más directamente ligadas con el sector agrícola, los informes insisten en la componente cultural que tienen cada una de estas prácticas como un "hecho social total" (Mauss, 1950), que refleja de forma más general, la cultura en la estas se enmarcan. El expediente griego citado anteriormente explica, por ejemplo, como en torno al cultivo y procesamiento de la resina de lentisco producido por éste, usada como aromatizante alimentario o en farmacología, se tejen redes de solidaridad y alianza y cómo la práctica, ya en el origen de las leyendas locales, es importante en el imaginario colectivo. La fuerte dimensión social de la preparación y consumo de alimentos se pone de relieve en varios casos. El dossier titulado "kimjang, preparar y compartir kimchi" (verduras fermentadas con especias), presentado por la República de Corea e inscrito en 2013, describe una práctica social cooperativa social estacional que reúne a una comunidad en la preparación de grandes cantidades de kimchi destinadas a durar todo el invierno y a ser compartida con los necesitados. Argumentos similares subyacen en la candidatura presentada por la República Popular Democrática de Corea con el nombre de "La tradición y la preparación de kimchi en la República Popular Democrática de Corea" y escrito dos años después de la primera. El informe titulado "Lavash: preparación, significado y la apariencia del pan tradicional armenio como expresión cultural", inscrito en 2014, describe la preparación altamente socializada de un alimento está presente en las comidas diarias como acompañamiento de carnes, verduras o queso en momentos solemnes, tales como bodas. Siempre resaltando su condición social dos años más tarde Azerbaiyán, Irán, Kazajstán, Kirguistán y Turquía han obtenido el registro del mismo elemento con el nombre de "La cultura de la fabricación y el intercambio de lavash pan aplastado, Katyrma, Jupka, Yufka". El expediente de "la cultura y la tradición del café turco", elemento inscrito en 2013, no sólo presenta una práctica social relacionada con el consumo de alimentos o bebidas, sino que insiste en el hecho de más allá de ser una bebida preparada con técnicas especiales, el café turco es una práctica colectiva alojada en espacios culturales específicos, que tiene una función de reunión social y se asocia con creencias populares arraigadas. En 2015 fue inscrito un elemento semejante, llamado "El café árabe, un símbolo de generosidad", descrito como un ritual de hospitalidad de los Emiratos Árabes Unidos, Arabia Saudita, Omán y Qatar. 
En 2016, tanto Tajikistan que Ouzbékistan han obtenido, por separado, el registro de su plato tradicional a base de arroz, carne, verduras y especias, llamado Oshi Palav y Palov respectivamente. En ambos casos, estos elementos se describen recalcando su función social y su relación con las ocasiones festivas o rituales. Bélgica ha hecho registrar la cultura de la cerveza, demostrando cómo este elemento une a unas comunidades amantes de la cerveza en el respeto del desarrollo sostenible y conscientes de los riesgos para la salud asociados al consumo de alcohol. En algunos casos, como en un día de fiesta de los viticultores de Vevey o del festival internacional de la cultura y de la Pesca de Argungu, candidaturas, respectivamente, de Suiza y Nigeria, estas nominaciones agrícolas ponen en el primer plano a los actos festivos asociados con las prácticas agrícolas o pesqueras.

Este breve panorama hace que sea posible extender algunas de las consideraciones hechas en el caso analizado con más detalle de la comida gastronómica de los franceses: por un lado, muestra el poder de las directivas técnicas performativas formuladas y vehiculadas por expertos internacionales en la construcción del patrimonio, construcción en estos casos coherente con las recomendaciones de la reunión de expertos de Vitré; por otra parte, llama la atención el intento de amortiguar, gracias a la aplicación de estas directivas, los componentes comerciales asociados con los alimentos.

Las formas de representar las prácticas agroalimentarias en relación con los actos festivos, rituales o conocimiento artesanales y hacer hincapié en su función social, sugerido por las directivas Vitré, han facilitado la entrada de las prácticas alimentarias en el ámbito de acción de la Convención para la salvaguarda del patrimonio cultural inmaterial. Estas directivas pueden ser consideradas como ejemplos del papel determinante que han desempeñado las directivas técnicas en la gestión global del patrimonio: su influencia es tanto directa, en el caso de la candidatura francesa, como indirecta, en la de Japón, que según Voltaire Cang (2015) habría sido creada siguiendo el ejemplo de la comida gastronómica de los franceses, convirtiéndose ésta en un modelo para otras candidaturas $\operatorname{alimentarias}^{28}$.

Como se ha señalado en otros ámbitos, la "cultura de las directivas" (Larsen 2013) redefine los objetivos políticos en términos técnicos y permite aplicar instrumentos de gobernanaza global legalmente poco vinculantes y darles un impacto real. En este caso, las directivas técnicas le permiten "empaquetar" el expediente de candidatura de los elementos del patrimonio cultural inmaterial a partir de realidades complejas y multidimensionales, compartirmentando aspectos culturales y económicos en la representación de las prácticas culturales candidatas a las listas de la Convención revistiendo así decisiones políticas, amenudo controvertidas, con una "pátina de la armonía" (Müller, 2013).

28. Por motivos de procedimiento, esta carta, sin embargo, no fue tomada en consideración por el Comité. 
Al igual que en el caso francés, la experiencia técnica es crucial para aplicar correctamente estas directivas. Por ejemplo, de acuerdo con uno de los expertos internacionales más activos en la difusión y aplicación de las directivas que la aplicación de la Convención, mi interlocutor, la candidatura de la cerveza belga ha sido aceptada porque quien redactó el documento ha sido capaz de encontrar "las palabras correctas" vaciándola de las problemáticas comerciales y haciendo un uso hábil del lenguaje utilizado en las directivas técnicas establecidas por los expertos de la Convención y su retórica. Por el contrario, la candidatura de Eslovenia "producción tradicional de salchicha de Cragno, propuesta para su inscripción en la Lista Representativa en 2015, fue evaluada negativamente por el organismo de evaluación debido a la insistencia en la certificación de la autenticidad de los procesos de producción, tutelados por una denominación de origen geográfica. Otro elemento de debilidad se atribuyó a la atención puesta en los productos en lugar de en las habilidades necesarias para su preparación o en su función social, atención que beneficia a los grupos de presión empresariales (UNESCO, 2015: 37-38). En este caso, el expediente se ha presentado sin depurar el lenguaje de los términos considerados inapropiados en virtud del Convenio y sin tener en cuenta las directivas técnicas que han permitido que otras candidaturas puedan prepararse de una manera considerada satisfactoria por el organismo de evaluación, autorizado a juzgar las candidaturas en base exclusivamente a la información contenida en los expedientes presentados por el Estado miembro. El hecho de no haber sido capaz de "ocultar las verdaderas intenciones de las partes interesadas", basadas en cuestiones esencialmente económicas, ha determinado, de acuerdo con el análisis de Raúl Matta, también el fracaso de la candidatura de la cocina peruana (2016: 350). Salta, de hecho, a los ojos de un observador del discurso de los expertos que han participado en la preparación o evaluación de los informe que "tener expedientes ganadores es sobre todo una cuestión de práctica" (Matta, 2016: 350) y, podemos añadir, de familiaridad con el lenguaje de Convención. Así, por ejemplo, la decisión de candidar "el arte de los pizzeros napolitanos" en lugar de la pizza, como en el proyecto inicial, depende de la experiencia de los expertos del Ministerio italiano de las políticas agrícolas, de su familiaridad con el espíritu de la Convención, del fino dominio de su lenguaje y de las recomendaciones que permiten representar las prácticas sociales o culturales como elementos del patrimonio cultural inmaterial. El nuevo nombre del elemento, de hecho, se centra en los procesos y prácticas de una comunidad en lugar de en un producto y el reconocimiento de la UNESCO se entiende como una oportunidad genérica para contrarrestar la pérdida de la diversidad biocultural (Petrillo, 2014). En general, los expedientes de elementos registrados presentan las prácticas asociadas a la alimentación de modo coherente con los principios de la Convención, a menudo haciendo hincapié en la componente cultural y la complejidad de los vínculos orgánicos entre muchos de ellos y la lógica del mercado. Este enfoque refleja la visión consolidada de que el patrimonio es un bien inalienable por excelencia (Fabre, 2016: 47) 
y sus relaciones con el mercado, que lo convierten en una simple mercancía, ponen en peligro su propia razón de ser, porque se convertiría en beneficio de pocos, en una simple mercancía (Settis, 2002). En algunos casos, esta opinión es compartida por algunos de los actores involucrados, como lo demuestran las alegaciones presentadas contra el proyecto de inscripción de la dieta mediterránea en una carta enviada a la UNESCO antes de la inclusión de este elemento. El discurso de los actores políticos y sociales, que se desprende de los documentos presentados a la UNESCO, insiste explícitamente en la importancia de la capacidad comercial asociada a la inscripción y considera el reconocimiento de la UNESCO como una marca de autenticidad que garantiza la autenticidad de sus prácticas, y por tanto de los productos resultantes. Por ello, los argumentos de los partidarios de la candidatura del 'arte de los pizzeros napolitanos", ampliamente difundidos en Internet, hacen referencia a un producto gastronómico: los partidarios del reconocimiento de la pizza como bien inmaterial, haciendo de la protección del "made in Italy" su objetivo, sitúan el proyecto en el ámbito comercial ${ }^{29}$. La motivación detrás de este compromiso es la lucha contra las imitaciones y falsificaciones y por lo tanto presupone la defensa de un producto original. Las entrevistas del promotor principal de esta candidatura, Alfonso Pecoraro Scanio, explican la necesidad de intervenir de cara a lo que el ex ministro ha llamado "la agropiratería. Es para contrarrestar este fenómeno, explica, que se dirigió inicialmente a la Organización Mundial del Comercio $(\mathrm{OMC})^{30}$. Los argumentos del ex ministro demuestran como la salvaguardia de los intereses comerciales italianos se basan en la valorización de la autenticidad de los productos. La solicitud de la marca europea de la Especialidad Tradicional Garantizada, dada en 2010 a la pizza napolitana, se explica precisamente por este enfoque de tutelar la "verdadera pizza". La Convención para la salvaguardia del patrimonio inmaterial es considerada por los actores políticos y sociales como un instrumento adecuado para salvaguardar los conocimientos y saberes denominados "tradicionales" que distinguen un producto "original" de los que simplemente están caracterizados por el llamado "italian sound", o lo que es lo mismo, del hecho de "sonar italiano" en los oídos de los consumidores internacionales.

El enfoque de la cuestión de la autenticidad de los métodos de producción, que en el sistema de Indicaciones Geográficas son inseparables de la dimensión territorial, permite resaltar las diferencias sustanciales entre esto último y la Convención para la salvaguardia del patrimonio inmaterial. Ni la autenticidad ni la asociación con una única zona geográfica son requisitos para el patrimonio inmaterial (Bortolotto 2011), y contrariamente a las Denominaciones Geográficas, la Convención UNESCO no garantiza ningún monopolio sobre las prácticas en cuestión (Kono, 2009). Sin embargo, las directivas operativas de la Convención, recientemente modificadas por la inclusión de un apartado dedicado al desarrollo sostenible, recomiendan a los estados de hacer

29. https://www.change.org/p/proteggiamo-il-made-in-italy-la-pizza-come-patrimonio-unesco 30. https://www.youtube.com/watch?v=vQnyZ7tGpJE 
cumplir los derechos de propiedad intelectual, incluidas las denominaciones de origen, para proteger a los depositarios del patrimonio cultural inmaterial en su actividades comerciales (UNESCO, 2016, art. 104 y 173).

El ámbito de la alimentación hace emerger con especial agudeza uno de los aspectos más complejos de la Convención. Por un lado, esta herramienta tiene objetivos puramente culturales, condena el abuso comercial del patrimonio y demuestra una gran prudencia en la evaluación de los efectos económicos derivados de las inscripciones en las listas internacionales de patrimonio inmaterial. Por otro, y en modo siempre más explícito, la Convención promueve la idea de una relación entre salvaguardia del patrimonio inmaterial y el llamado desarrollo sostenible, portador también de beneficios económicos para las comunidades involucradas, a menudo en estrecha relación con un territorio. Esta tensión es la fuente de gran controversia durante las reuniones del Comité. Un ejemplo es el que se dió con motivo de la candidatura de Kirguistán "Ala-kiyiz y Shyrdak, arte de la alfombra tradicional de Kirguistán en fieltro". El expediente de la solicitud propone medidas para desarrollar la industria de las alfombras para hacer frente a las amenazas que pesan sobre esta práctica: la falta de interés de las nuevas generaciones y la difusión de las alfombras sintéticas industriales. La opinión contraria a la inscripción expresada por el órgano de evaluación fue motivada por la excesiva concentración de medidas de salvaguardia en la promoción económica de la práctica. La delegación de Kirguistán, sin embargo, defendió con éxito su candidatura alegando que los fuertes lazos con el mercado son la única manera de hacer sobrevivir los conocimientos artesanales y que el vínculo con la economía local no disminuye el valor cultural de las prácticas artesanales. Desde esta perspectiva, que se encuentra en perfecta consonancia con el discurso militante del empoderamiento a través de la afirmación de la identidad y la valorización del capital étnico (Comaroff y Comaroff, 2009), se reivindica la complementariedad de los aspectos económicos y culturales y la comercialización de productos artesanales se entiende como una forma de desarrollo sostenible y de economía creativa ${ }^{31}$ (31).

\section{CONCLUSIONES}

El campo de los bienes inmateriales alimentarios es hoy día un sector importante en la aplicación de la Convención para la salvaguardia del patrimonio cultural inmaterial a pesar de que no estaba entre los previstos inicialmente en el curso de las negociaciones en el origen de este instrumento. Las candidaturas de elementos agroalimentarios son juzgadas con precaución por los posibles abusos comerciales relacionados con su valorización. En la primera fase de la aplicación de la Convención, las directivas de la UNESCO alentaron las representaciones esencialmente culturales de las prácticas alimentarias candidadas a las listas de la Convención. Desde esta perspectiva, aislar la dimensión patrimonial de las

31. Notas de campo. 
prácticas alimentarias para representarlas como elementos salvaguardables en el ámbito de la Convención de la UNESCO, a menudo ha demostrado ser una tarea difícil para los actores políticos y sociales que tienen, sin embargo, una visión holística. La aplicación internacional de este instrumento está, no obstante, fomentando un vínculo siempre más explícito entre la salvaguardia del patrimonio inmaterial y el desarrollo sostenible. Dada la relación directa entre las prácticas alimentarias, el uso de los recursos naturales y el mercado, los bienes inmateriales relacionados con el ámbito agroalimentario se ven particularmente afectados por esta evolución del espíritu de la Convención. Las prácticas alimentarias se encuentran en elámbito de aplicación de variosinstrumentos de protección, como las denominaciones de origen, o la Convención para la protección y promoción de la diversidad de las expresiones culturales, además, obviamente, de la Convención para la salvaguardia del patrimonio cultural inmaterial (Herrero de la Fuente, 2009; Broude, 2015). La coordinación de estos instrumentos, cuyos principios respectivos puede entrar en conflicto entre sí, está creando nuevos esquemas de patrimonialidad para la protección de la diversidad biológica y cultural que aún no se han explorado. De hecho, diversos elementos inscritos en las listas internacionales del PCI por la UNESCO están protegidos también por indicaciones geográficas (Ubertazzi, en prensa). Si ambos dispositivos usan de modo similar el discurso sobre la tradición para crear un valor añadido (Aguilar, Amaya y López, 2016), esos se basan sobre presupuestos diversos, en particular sobre un diverso modo de concebir la relación entre cultura y territorio, y son independientes los unos de los otros. A pesar de la heterogeneidad de los instrumentos utilizados para promover los bienes culturales alimentarios, este escenario muestra cómo los bienes intangibles relacionados con el ámbito agroalimentario ofrecen un marco de acción ideal para el "emprendimiento patrimonial" (Pfeilstetter, 2015) y encarnan por antonomasia el carácter proactivo de la gestión patrimonial contemporánea, ya no circunscrita a la mera conservación sino dirigida a la promoción de posibles usos culturales, sociales y económicos (Matta, 2015). Utilizado como un recurso para ser invertido con objetivos socio-políticos o económicos, el patrimonio se considera cada vez más un ingrediente clave del llamado desarrollo sostenible (Yúdice, 2003: 6-7) ilustrando el controvertido papel que desempeña la cultura en la era de la globalización neoliberal. 


\section{REFERENCIAS BIBLIOGRÁFICAS}

Bortolotto, Chiara (2011) "Il patrimonio immateriale e l'autenticità: una relazione indissolubile". La Ricerca Folklorica 64, pp. 7-17.

Broude, Tomer (2015) "A Diet Too Far? Intangible Cultural Heritage, Cultural Diversity, and Culinary Practices". En Calboli, Irene y Ragavan, Srividhya (coords.) Diversity in Intellectual Property: Identities, Interests, and Intersections. Cambridge: Cambridge University Press, pp. 472-493.

Cang, Voltaire (2015) "Unmaking Japanese Food: Washoku and Intangible Heritage Designation”. Food Studies: An Interdisciplinary Journal 5(3), pp. 49-58.

Chevrier, Francis (2011a) Notre gastronomie est une culture. Paris : François Bourin.

Chevrier, Francis (2011b) "Notre gastronomie est une culture», de Francis Chevrier : manger, un patrimoine". Le Monde, 10/06/2011 http://www.lemonde. fr/livres/article/2011/06/10/notre-gastronomie-est-une-culture-de-francischevrier_1534501_3260.html\#Oeoh4xsghWLzr6sV.99 [Consultado el 18 de noviembre 2016].

Comaroff, Jean y Comaroff, John (2009) Ethnicity, Inc. Chicago : University of Chicago Press.

Aguilar Criado, Encarnación, Amaya Corchuelo, Santiago y López Moreno, Ignacio (2016) "Alimentos con calidad. Nuevas estrategias rurales para nuevos consumidores". Revista Arxiu d'Etnografia de Catalunya 16, pp.137-152.

Csergo, Julia (2011) “Le 'Repas gastronomique des Français' à l'Unesco : éléments d'une inscription au patrimoine culturel immatériel de l'humanité ». OCHA, online : $\underline{w w w}$. lemangeur-ocha.com [Consultado el 15 de novembre 2016].

Csergo, Julia (2016) La gastronomie est-elle une marchandise culturelle come une autre? La gastronomie française à l'Unesco: Histoire et enjeux. Chartres : Menu Fretin.

Detienne, Marcel (2010) L'identité nationale, une énigme. Paris: Gallimard.

Fabre, Daniel (2016) "Lordinaire, le familier, l'intime...loins du monument". En Christian,. Hottin y Voisenat, Claudie (coords.) Le tournant patrimonail. Mutations contemporaines des méties du patrimoine. Paris: Éditions de la Maison des sciences de l'homme, pp. 43-58.

Grillo, Carmine (2008) "Come mangiarsi un Patrimonio mondiale. I Sassi di Matera”. Il Resto 6 (4), p. 6.

Hafstein, Valdimar (2008) "Inviting a Noisy Dance-Band into a Hospital: Listing the Intangible”. En Chiara Bortolotto (coord..) Il patrimonio immateriale secondo l'Unesco : analisi e prospettive. Roma: Istituto Poligrafico e Zecca dello Stato, pp. 95-113. 
Hartog, François (2003) Régimes d'historicité. Présentisme et expérience du temps. Paris: Seuil.

Heinich, Nathalie (2009) La fabrique du patrimoine. "De la cathédrale à la petite cuillère". Paris: Éditions de la Maison des sciences de l'homme.

Herrero de la Fuente, Alberto A. (2009) “La Convención dela UNESCO para la salvaguardia del patrimonio cultural inmaterial de 17 de octubre de 2003 y las indicaciones de calidad de los alimentos". En Benedetta Ubertazzi y Esther Muñiz (coords.) Le indicazioni di qlualità degli alimenti. Diritto internazionale ed europeo. Milano: Giuffré, pp. 11-26.

IEHCA, (s.d.) Pour l'Inscription par l'Unesco du patrimoine alimentaire français sur la liste représentative du patrimoine culturel immatériel de l'humanité.

Jeudy, Henri-Pierre (2001) La machinerie patrimoniale. Paris: Sens \& Tonka.

Kirshenblatt-Gimblett, Barbara (2004) "Intangible cultural heritage as a meta-cultural production”. Museum International 221-222, pp. 53-66.

Kono, Toshiyuki (2009) "Geographical Indications and Intangible Cultural Heritage". En Benedetta Ubertazzi y Esther Muñiz (coords.) Le indicazioni di qlualità degli alimenti. Diritto internazionale ed europeo. Milano: Giuffré, pp. 11-299.

Larsen, Peter Bille (2013) “The politics of technicality: Guidance culture in environmental governance". En Birgit Müller (coord.) The gloss of harmony: The politics of policymaking in multilateral organisations. London: Pluto, pp. 75-100.

Levi, Carlo (1945) Cristo si è fermato ad Eboli. Torino : Einaudi.

Lowenthal, David (1996) Possessed by the past: the heritage crusade and the spoils of history. New York: The Free Press.

Marques da Silva, António José (2016) Diaita Nostra. Patrimoines alimentaires, identité et gouvernementalité en Méditerranée. Évora : UNESCO Chair in intangible heritage and traditional know-how: linking heritage - Évora University. http://www.catedra.uevora. pt/unesco/index.php/unesco/Investigacao/Publications-et-al/Books/Diaita-NostraPatrimoines-alimentaires-identite-et-gouvernementalite-en-Mediterranee [Consultado el 18 de desembre 2016].

Matta, Raúl (2015) "Edible Identities: Food as Cultural Heritage by Ronda L. Brulotte and Michael A. Di Giovine (Eds.)". Anthropology of food http://aof.revues.org/7824

[Consultado el 13 de octubre 2016].

Matta, Raúl (2016) "Food incursions into global heritage: Peruvian cuisine’s slippery road to UNESCO”. Social Anthropology 24 (3), pp. 338-352.

Mauss, Marcel (1950) [1923-1924] "Essai sur le don. Forme et raison de l'échange dans les sociétés archaïques”. En Sociologie et Anthropologie. Paris: PUF, pp. 143-279. 
Müller, Birgit (2013) "Introduction: Lifting the veil of harmony: Anthropologists approach international organizations". En Birgit Müller (coord.) The Gloss of Harmony: The Politics of Policy Making in Multilateral Organisatios. London : Pluto Press, pp.1-20. Murphy, Cullen (2001) “Immaterial civilization". The Athlantic Monthly 288 (2), pp. $20-$ 21.

Nas, Peter J.M. (2002) "Masterpieces of oral and intangible culture. Reflections on the UNESCO World Heritage List”. Current Anthropology 43(1), pp. 139-148.

Petrillo, Pier Luigi (2014) Sostenibilita' ambientale e patrimonio agro-alimentare. Intervento al Salone del gusto - Terra madre, Torino, 24 novembre 2014.

Pfeilstetter, Richard (2015) "Heritage entrepreneurship. Agency-driven promotion of the Mediterranean diet in Spain". International Journal of Heritage Studies 21(3), pp. 215231.

Sammells, Clare A. (2014) “Haute Traditional Cuisines: How UNESCO’s List of Intangible Heritage Links the Cosmopolitan to the Local". En Ronda Brulotte y Michael Di Giovine (coords.) Edible Identities: Food as Cultural Heritage. Farnham: Ashgate, pp. 141-158. '

Settis, Salvatore (2002) Italia S. p. A. Torino : Einaudi.

Steinberger, Michael (2009) Au Revoir To All That: Food, Wine, and the End of France. New York : Bloomsbury.

Tornatore, Jean-Louis (2012) "Anthropology's Payback: 'The Gastronomic Meal of the French. The Ethnographic Elements of a Heritage Distinction". En Regina Bendix, Aditya Eggert y Arnika Peselmann (coords.), Heritage Regimes and the State. Göttingen: Universitätsverlag Göttingen, pp. 341-365.

Ubertazzi, Benedetta (en prensa) "EU Geographical Indications and Intangible Cultural Heritage". International Review of Intellectual Property and Competition Law.

UNESCO, (2003) Convention for the Safeguarding of the Intangible Cultural Heritage. UNESCO, (2005) Convention on the Protection and Promotion of the Diversity of Cultural Expressions.

UNESCO, (2009) Summary records of the committee's third ordinary session (ITH/09/4. COM/CONF.209/5).

UNESCO, (2010) Convention for the safeguarding of the intangible cultural heritage. Intergovernmental committee for the safeguarding of the intangible cultural heritage, fifth session, Nairobi, Kenya, November 2010, nomination file no. 00437 for inscription on the representative list of the intangible cultural heritage in 2010. 
UNESCO, (2011) Adoption of the summary records of the fifth session of the Committee (ITH/11/6.COM/CONF.206/4 Rev.).

UNESCO, (2015) Convention for the safeguarding of the intangible cultural heritage. Intergovernmental committee for the safeguarding of the intangible cultural heritage. Tenth session Windhoek, Namibia 30 November to 4 December 2015. Item 10.b of the Provisional Agenda: Examination of nominations for inscription on the Representative List of the Intangible Cultural Heritage of Humanity.

UNESCO, (2016) Operational Directives for the Implementation of the Convention for the Safeguarding of the Intangible Cultural Heritage.

Yüdice, George (2004) The Expediency of Culture: Uses of Culture in the Global Era. Durham: Duke University Press. 